\title{
HAM Solution of an Unsteady Three Dimensional Navier-Stokes Equations of Flow between Two Parallel Disks
}

\section{Selvarani $\mathbf{S}^{*}$ and Nithyadevi $\mathbf{N}$}

Department of Mathematics, Bharathiar University, Coimbatore, Tamilnadu, India

\begin{abstract}
Purpose: The paper aims to find an analytic solution of an unsteady three di-mensional Navier-Stokes equations of flow between two parallel disks by using von Karman type similarity transformation.

Methodology: In this paper, the Homotopy Analysis Method (HAM) with the value of unknown convergence control parameter has been used to derive accurate analytic solution for an unsteady three dimensional NavierStokes equations of flow between two parallel disks. The possible optimal value of the convergence control parameter determined by increasing the order of HAM.
\end{abstract}

Findings: The results obtained from HAM are compared with numerical results. The result shows that this method gives an analytic solution with high order of accuracy with a few iterations.

Keywords: 3D Navier-Stokes equation; Homotopy analysis method; Convergence; System of nonlinear differential equations

\section{Introduction}

In mathematics and physics, nonlinear partial differential equations are partial differential equations with nonlinear terms. A few nonlinear differential equations have known exact solutions, but many which are important in applications do not. Sometimes these equations may be linearized by an expansion process in which nonlinear terms are discarded. When nonlinear terms make vital contributions to the solution this cannot be done, but sometimes it is enough to retain a few small ones. Then a perturbation theory may be used to obtain the solution. The differential equations may sometimes be approximated by an equation with small nonlinearities in more than one way, giving rise to different solutions valid over different range of its parameters.

Most scientific and engineering problems are modeled by ordinary differential equations or partial differential equations, some of them are solved using the analytic methods of perturbation by Nayfeh [1]. In the numerical methods, stability and convergence should be considered so as to avoid divergence or inappropriate results. In the analytic perturbation methods, we should exert the small parameter in the equation. In numerical methods the advantage is that we have to use the small parameter a lot since most problems do not have known analytic solutions or that if they are known it is too complex to deal with them. The main advantage in analytic method is that it is exact and gives us more contexts. One of the semi-exact methods which do not need small or large parameters is the Homotopy Analysis Method (HAM), first proposed by Liao in his thesis. Liao [2] employed the basic ideas of homotopy in topology to propose a general analytic method for nonlinear problems, namely HAM, which is a powerful analytical method for solving linear and nonlinear differential equations. The HAM also avoids discretization and provides an efficient solution with high accuracy, minimal calculations and avoidance of physically unrealistic assumption. Furthermore, the HAM always provides us with a family of solution expressions with the auxiliary parameter $\sim$, the convergence region and the rate of each solution might be determined conveniently by the auxiliary parameter $\hbar$. HAM contains the homotopy perturbation method (HPM) discussed by He [3], the Adomian decomposition method (ADM) examined by Allan [4], and the d-expansion method.
The main goal of the present study is to find the totally analytic solution for the problem of flow between two disks by the HAM. In this way, the paper has been organized as follows. In Chapter 2, the flow analysis and mathematical formulation are presented. We extend the application of the HAM to construct the approximate solutions for the governing equations. And we have analyzed the convergence of the obtained series solutions. Chapter 3 contains the results and discussion. Finally conclusions are summarized.

\section{Mathematical Formulation}

Consider the axis-symmetric flow between two in finite disks with a distance $\mathrm{d}$ between them. Both disks are placed in the radial direction with a velocity proportional to the radii. The bottom disk is located in the $\mathrm{z}=0$ plane. The velocity ratio of the upper disk to the lower one is $\gamma$ and $\epsilon$ is the amplitude of the disk. For an incompressible fluid without body forces and based on axis symmetric reads from the papers discussed by Dinarvand [5].

$$
\begin{aligned}
& \frac{1}{r} \frac{\partial}{\partial r}\left(r u_{r}\right)+\frac{\partial u_{z}}{\partial z}=0, \\
& \frac{\partial u_{r}}{\partial t}+u_{r} \frac{\partial u_{r}}{\partial r}+u_{z} \frac{\partial u_{r}}{\partial z}=v\left(\frac{\partial^{2} u_{r}}{\partial r^{2}}+\frac{1}{r} \frac{\partial u_{r}}{\partial r}+\frac{\partial^{2} u_{r}}{\partial z^{2}}-\frac{u_{r}}{r^{2}}\right), \\
& \frac{\partial u_{z}}{\partial t}+u_{r} \frac{\partial u_{z}}{\partial r}+u_{z} \frac{\partial u_{z}}{\partial z}=v\left(\frac{\partial^{2} u_{z}}{\partial r^{2}}+\frac{1}{r} \frac{\partial u_{z}}{\partial r}+\frac{\partial^{2} u_{z}}{\partial z^{2}}\right) .
\end{aligned}
$$

Where the velocity vector $\bar{V}=\left(u_{r}, u_{z}\right)$, v is the kinematic viscosity.

*Corresponding author: Selvarani S, Department of Mathematics, Bharathiar University, Coimbatore-641 046, Tamilnadu, India, Tel: 914222605162; E-mail s.selvarani91@gmail.com.

Received September 02, 2015; Accepted September 14, 2015; Published September 18, 2015

Citation: Selvarani S, Nithyadevi N (2015) HAM Solution of an Unsteady Three Dimensional Navier-Stokes Equations of Flow between Two Parallel Disks. J Appl Computat Math 4: 256. doi:10.4172/2168-9679.1000256

Copyright: (c) 2015 Selvarani S, et al. This is an open-access article distributed under the terms of the Creative Commons Attribution License, which permits unrestricted use, distribution, and reproduction in any medium, provided the original author and source are credited. 
By using von Karman type similarity transformations, similarity functions can be sought as follows,

$$
u_{r}=r F(\eta) \text {, and } u_{z}=d H(\eta)
$$

Where $\eta=\frac{z}{d}=\frac{z}{\gamma t}$ is the similarity variable. Substituting the similarity functions into the equations (1), (2) and (3). Therefore, the governing equations yields a similarity equation group

$$
\left\{\begin{array}{llc}
F^{\prime \prime} & = & \operatorname{Re}\left(F^{2}+R e F+2 R e H F+R e H^{2} F\right) \\
H^{\prime}= & -2 F
\end{array}\right.
$$

with boundary conditions

$$
F(0)=1+\epsilon \text { cost }, \quad H(0)=H(1)=0 \text { and } \quad F(1)=\gamma \text {. }
$$

Where $R e=\frac{d^{2}}{v}$ is the Reynolds number of the wall and $\gamma$ is the parameter of the upper disk showing the velocity ratio of the upper disk to the bottom disk. Without loss of generality, we assumed that $0 \leq \gamma \leq 1$.

\section{Analytical Solution with HAM}

Due to basic idea of HAM, as described in detail by Liao [6], according to the boundary conditions (5), we choose

$$
\begin{aligned}
& F_{0}(\eta)=1-(1+\epsilon \cos t-\gamma) \eta+\epsilon \cos t, \\
& H_{0}(\eta)=0,
\end{aligned}
$$

as initial guesses of $\mathrm{F}(\eta)$; and $\mathrm{H}(\eta)$ which satisfy the boundary conditions (5). Besides, we select the auxiliary linear operators L1(F); and $\mathrm{L} 2(\mathrm{H})$ as

$$
\begin{aligned}
& L_{1}(F)=F^{\prime \prime}, \\
& L_{2}(H)=H^{\prime},
\end{aligned}
$$

satisfying the following properties

$$
\begin{aligned}
& \mathrm{L}_{1}\left(\mathrm{C}_{1} \eta+\mathrm{C}_{2} \eta\right)=0, \\
& \mathrm{~L}_{2}\left(\mathrm{C}_{3}\right)=0 .
\end{aligned}
$$

where $c_{i} ; i=1 ; 2 ; 3$ are arbitrary constants. If $q 2[0 ; 1]$ is an embedding parameter and $\hbar$ is an auxiliary nonzero parameter, then the zerothorder deformation equations are of the following form,

$$
\begin{aligned}
& (1-q) \mathcal{L}_{1}\left[\hat{F}(\eta ; q)-F_{0}(\eta)\right]=q \hbar \mathcal{N}_{1}[\hat{H}(\eta ; q), \hat{F}(\eta ; q)], \\
& (1-q) \mathcal{L}_{2}\left[\hat{H}(\eta ; q)-H_{0}(\eta)\right]=q \hbar \mathcal{N}_{2}[\hat{H}(\eta ; q), \hat{F}(\eta ; q)] .
\end{aligned}
$$

subject to the boundary conditions

$$
\begin{aligned}
& \hat{F}(0 ; q)=1+\epsilon \text { cost }, \quad \hat{F}(1 ; q)=\gamma, \\
& \hat{H}(0 ; q)=\hat{H}(1 ; q)=0,
\end{aligned}
$$

in which we define the nonlinear operators $\mathrm{N}_{1}$; and $\mathrm{N}_{2}$ as

$$
\begin{aligned}
& \mathcal{N}_{1}[\hat{H}(\eta ; q), \hat{F}(\eta ; q)]= \frac{\partial^{2} \hat{F}(\eta ; q)}{\partial \eta^{2}}-\operatorname{Re}(2 \operatorname{Re}(\hat{H}(\eta ; q))(\hat{F}(\eta ; q))+\operatorname{Re}(\hat{F}(\eta ; q)) \\
&+(\hat{F}(\eta ; q))^{2}+\operatorname{Re}(\hat{H}(\eta ; q))^{2}(\hat{F}(\eta ; q)), \\
& \mathcal{N}_{2}[\hat{H}(\eta ; q), \hat{F}(\eta ; q)]=\frac{\partial \hat{H}(\eta ; q)}{\partial \eta}+2 \hat{F}(\eta ; q) .
\end{aligned}
$$

Clearly, when $\mathrm{q}=0$ the zero-order deformation equations (12) and (13) give rise to:

$$
\hat{F}(\eta ; 0)=F_{0}(\eta), \hat{H}(\eta ; 0)=H_{0}(\eta), \hat{P}(\eta ; 0)=P_{0}(\eta) .
$$

when $\mathrm{q}=1$, they become:

$$
\hat{F}(\eta ; 1)=F(\eta), \hat{H}(\eta ; 1)=H(\eta), \hat{P}(\eta ; 1)=P(\eta) \text {. }
$$

As q increases from 0 to $1, \hat{F}(\eta ; q)$ and $\hat{H}(\eta ; q)$ vary from $\mathrm{F}_{0}(\eta)$ and $\mathrm{H}_{0}(\eta)$ to $\mathrm{F}(\eta)$ and $\mathrm{H}(\eta)$. Expanding $\hat{F}(\eta)$ and $\hat{H}(\eta)$ in Maclaurin series with respect to the embedding parameter $\mathrm{q}$ and equations (14) and (15), we obtain

$$
\begin{aligned}
& \hat{F}(\eta ; q)=F_{0}(\eta)+\sum_{m=1}^{+\infty} F_{m}(\eta) q^{m}, \\
& \hat{H}(\eta ; q)=H_{0}(\eta)+\sum_{m=1}^{+\infty} H_{m}(\eta) q^{m},
\end{aligned}
$$

Where

$$
\begin{aligned}
& F_{m}(\eta)=\left.\frac{1}{m !} \frac{\partial^{m} \hat{F}(\eta, q)}{\partial q^{m}}\right|_{q=0}, \\
& H_{m}(\eta)=\left.\frac{1}{m !} \frac{\partial^{m} \hat{H}(\eta, q)}{\partial q^{m}}\right|_{q=0} .
\end{aligned}
$$

As pointed by Liao, the convergence of the series (16) and (17) strongly depend upon auxiliary parameter $\hbar$ [7-18]. Assume that $\hbar$ is selected such that the series (16) and (17) are convergent at $\mathrm{q}=1$ then due to equations (14) and (15) we have

$$
\begin{aligned}
& F(\eta)=F_{0}(\eta)+\sum_{m=1}^{+\infty} F_{m}(\eta), \\
& H(\eta)=H_{0}(\eta)+\sum_{m=1}^{+\infty} H_{m}(\eta) .
\end{aligned}
$$

Differentiating the zero-order deformation equations (12) and (13) $\mathrm{m}$ times with respect to $\mathrm{q}$, then setting $\mathrm{q}=0$ and finally dividing by $\mathrm{m}$ ! we have the $\mathrm{m}^{\text {th }}$-order deformation equations:

$$
\begin{aligned}
& \mathcal{L}_{1}\left[F_{m}(\eta)-\chi_{m} F_{m-1}(\eta)\right]=\hbar R_{1, m}(\eta), \\
& \mathcal{L}_{2}\left[H_{m}(\eta)-\chi_{m} H_{m-1}(\eta)\right]=\hbar R_{2, m}(\eta) .
\end{aligned}
$$

with the following boundary conditions

$\mathrm{F}_{\mathrm{m}}(0)=\mathrm{F}_{\mathrm{m}}(1)=0$ and $\mathrm{H}_{\mathrm{m}}(0)=\mathrm{H}_{\mathrm{m}}(1)=0$,

Where

$$
\begin{aligned}
R_{1, m}(\eta)= & \frac{\partial^{2} F_{m-1}(\eta)}{\partial \eta^{2}}-\operatorname{Re} \sum_{i=0}^{m-1}\left(F_{i}(\eta) F_{m-1-i}(\eta)+\operatorname{Re}_{i}(\eta)\right. \\
& +2 \operatorname{Re}_{i}(\eta) F_{m-1-i}(\eta)+\sum_{l=0}^{i} \operatorname{ReH}_{i}(\eta) H_{i-1}(\eta) F_{m-1-i}(\eta), \\
R_{2, m}(\eta) & =\frac{\partial H_{m-1}(\eta)}{\partial \eta}+2 F_{m-1}(\eta),
\end{aligned}
$$

and

$\chi_{m}= \begin{cases}0 & m \leq 1, \\ 1 & m>1 .\end{cases}$

Then the solutions for equations (20) and (21) can be expressed by: $F_{m}(\eta)=\chi_{m} F_{m-1}+\hbar \mathcal{L}_{1}^{-1}\left[R_{1, m}\right]+c 1 \eta+c 2$,

$H_{m}(\eta)=\chi_{m} H_{m-1}+\hbar \mathcal{L}_{2}^{-1}\left[R_{2, m}\right]+c 3$. 
where $\mathrm{c} 1 ; \mathrm{c} 2$; $\mathrm{c} 3$ are integral constants can be found by boundary conditions (22). For example, we can obtain the following result for solving the first-order deformation equation by using symbolic software MATHEMATICA, and successively obtain,

$$
\begin{aligned}
F_{1}(\eta)= & \frac{-1}{24} \hbar(-1+n) n \operatorname{Re}\left(9-9 n+3 n^{2}+8 \operatorname{Re}-4 n \operatorname{Re}+4 v+4 n v-4 n^{2} v\right. \\
& +4 \operatorname{Rev}+4 n \operatorname{Rev}+2 V^{2}+2 n v^{2}+2 n^{2} v^{2}-4(-3-2 \operatorname{Re}+n(3+\operatorname{Re}-v) \\
& \left.\left.+n^{2}(-1+v)-v\right) \operatorname{Cos}[t]+\left(3-3 n+n^{2}\right) \operatorname{Cos}[2 t]\right), \\
H_{1}(\eta)= & \hbar\left(-3+2 n+n^{2}(-1+v)+v-\left(3-2 n+n^{2}\right) \operatorname{Cos}[t] .\right.
\end{aligned}
$$

\section{Convergence of HAM Solution}

The totally analytic series solutions of the functions $F(\eta)$ and $\mathrm{H}(\eta)$ are given in equations (18 and 19). The convergence of these series and the rate of approximation for the HAM strongly depend upon the value of the auxiliary parameter $\hbar$, as pointed out by Liao [7]. In general, by means of the $\hbar$-curve, it is straightforward to choose a proper value of $\hbar$ to control the convergence of the approximation series [19-26]. To find the range of the admissible values of $\hbar, \hbar$-curves of $\mathrm{F}^{\prime \prime}(0)$ and $\mathrm{H}^{\prime}$ (0) obtained by the $11^{\text {th }}$ order approximation of the HAM for $\gamma=0$ and $\gamma=1$ at $\operatorname{Re}=1 ; t=1$ and $\epsilon=1$ are plotted in Figures 1 and 2, respectively. From these figures, the valid regions of $\hbar$ correspond to the line segments nearly parallel to the horizontal axis. Sometimes this region is not perfectly flat to the slowly convergence rate of the series solution. However a value of $\mathrm{h}$ can be picked up. Therefore for our problem we choose $\hbar=0: 4[27,28]$.

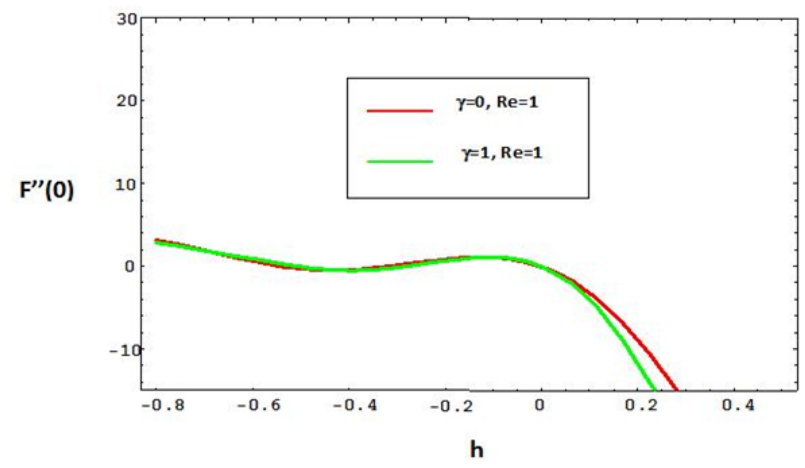

Figure 1: The h-curve of $F^{\prime \prime}(0)$, obtainedby $11^{\text {th }}$ order approximation of the HAM for $\mathrm{y}=0, \mathrm{y}=1$ and $\mathrm{t}=1$ with $\mathrm{Re}=1$.

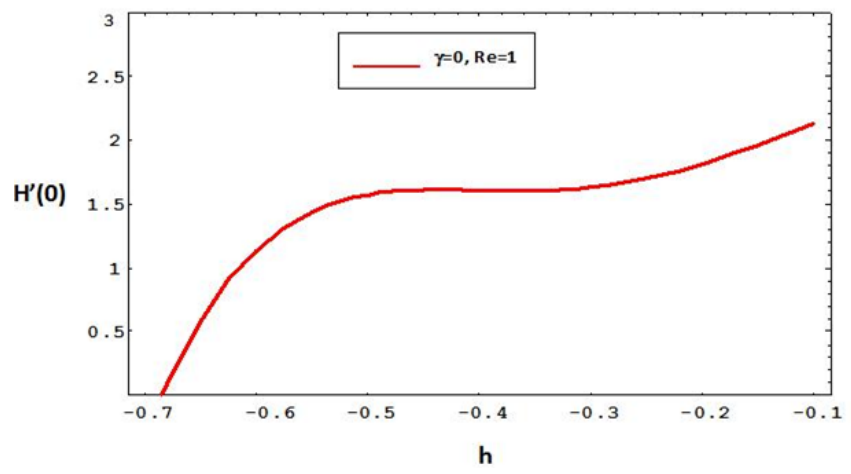

Figure 2: The h-curve of $H^{\prime \prime}(0)$, obtained by 11 th order approximation of the HAM for $y=0, y=1, \epsilon=0$ and $t=1$ with $R e=1$.

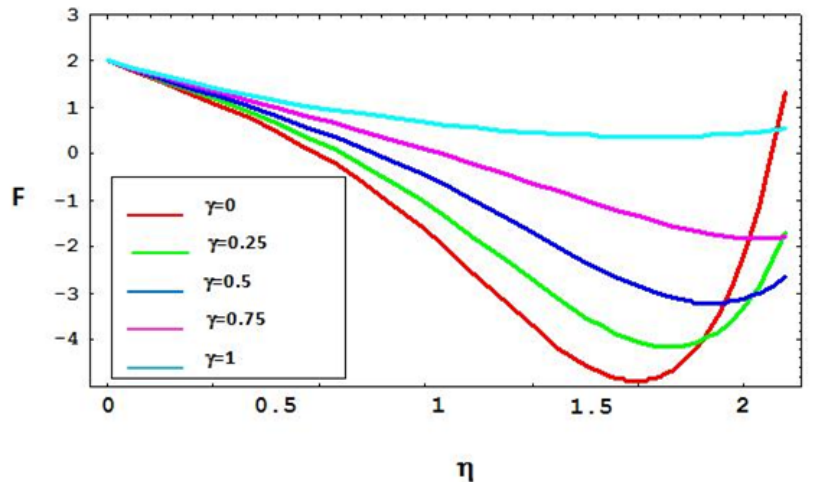

Figure 3: The velocity profiles in the radial direction obtained by the 11th order approximation of the HAM when $\epsilon=1, t=1$ and $R e=1$.

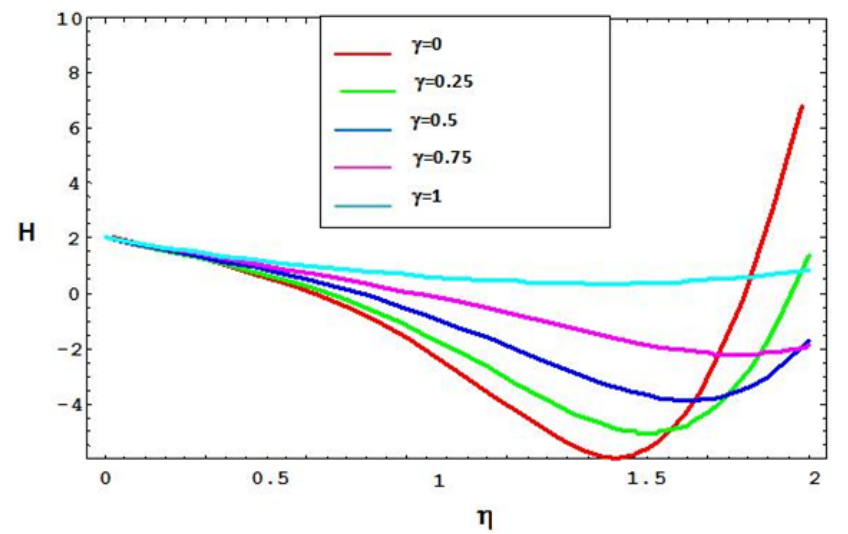

Figure 4: The velocity profiles in the vertical direction obtained by the $11^{\text {th }}$ order approximation of the HAM when $\epsilon=1, t=1$ and $R e=1$.

\section{Results and Discussion}

We studied the axis-symmetric flow between two infinite parallel disks and the results obtained from HAM solution are compared with numerical results. HAM method gives an analytic solution with high order of accuracy with small number of iterations. Graphical representation of results and tables are useful means for demonstrating the efficiency and accuracy of the HAM for the above problem. The velocity pro les in the radial direction obtained by the $11^{\text {th }}$ order approximation of the HAM for $\mathrm{Re}=1$, time and amplitude is to be constant with different values of $\gamma$ are shown in Figure 3.

For the radial flow velocity, it is found that the velocity near the wall is extended by the wall movement. However, away from the wall, there exists an out flow in the positive radial direction to balance the mass strained out by the wall to be consistent within the force balance. For $\gamma \neq 0$, the fluid is moved away from the wall with an outer flow in the radial direction near the lower disk.

Figure 4, shows that the velocity pro les in the vertical direction obtained by the $11^{\text {th }}$ order approximation of the HAM for $\mathrm{Re}=1$, time and amplitude is to be constant with different values of $\gamma$. The vertical velocity is downward near the bottom wall due to the movement of the wall. So the overall net flow rate is downward to balance the moving of the bottom disk as seen from the velocity profiles dominant for $(\gamma<1)$. For $\gamma \neq 1$, the net flow in the vertical direction is zero. The maximum negative velocity in the vertical direction decreases with the decreasing values of $\gamma$. 
Citation: Selvarani S, Nithyadevi N (2015) HAM Solution of an Unsteady Three Dimensional Navier-Stokes Equations of Flow between Two Parallel Disks. J Appl Computat Math 4: 256. doi:10.4172/2168-9679.1000256

Page 4 of 6

From Figures 3 and 4, we can observe a very good agreement between the analytic results of the HAM and numerical results shown in Tables 1 and 2 .

\begin{tabular}{|c|c|c|c|c|c|c|}
\hline $\mathbf{r}$ & $\eta$ & $9^{\text {th }}$ order & $10^{\text {th }}$ order & $11^{\text {th }}$ order & Numerical & Error \\
\hline \multirow{5}{*}{0} & 0 & +2.1619 & +2.1732 & +2.1736 & +2.1735 & -0.0001 \\
\hline & 0.5 & +1.1219 & +1.1328 & +1.1432 & +1.1431 & -0.0001 \\
\hline & 1 & -2.6529 & -2.7324 & -2.7632 & -2.7622 & 0.001 \\
\hline & 1.5 & -5.6405 & -5.6329 & -5.6432 & -5.6431 & 0.0001 \\
\hline & 2 & +4.6714 & +4.7325 & +4.7423 & +4.7422 & -0.0001 \\
\hline \multirow{5}{*}{0.25} & 0 & +2.5362 & +2.4563 & +2.4536 & +2.4542 & 0.0006 \\
\hline & 0.5 & +1.2005 & +1.2345 & +1.2346 & +1.2453 & 0.0107 \\
\hline & 1 & -2.6432 & -2.5634 & -2.5638 & -2.5636 & 0.0002 \\
\hline & 1.5 & -4.6532 & -4.6643 & -4.6743 & -4.6744 & -0.0001 \\
\hline & 2 & +1.6985 & +1.7051 & +1.7132 & +1.7325 & 0.0193 \\
\hline \multirow{5}{*}{0.5} & 0 & +2.3648 & +2.4563 & +2.4632 & +2.4621 & -0.0011 \\
\hline & 0.5 & +1.2795 & +1.2736 & +1.2836 & +1.2826 & -0.001 \\
\hline & 1 & -2.5632 & -2.5348 & -2.5448 & -2.5438 & 0.001 \\
\hline & 1.5 & -4.3624 & -4.4621 & -4.4632 & -4.4630 & 0.0002 \\
\hline & 2 & +1.8574 & +1.8795 & +1.8685 & +1.8683 & -0.0002 \\
\hline \multirow{5}{*}{0.75} & 0 & +2.4321 & +2.5464 & +2.5463 & +2.5645 & 0.0182 \\
\hline & 0.5 & +1.3562 & +1.3563 & +1.3654 & +1.3655 & 0.0001 \\
\hline & 1 & +1.9321 & +1.9465 & +1.9466 & +1.9465 & -0.0001 \\
\hline & 1.5 & -1.6959 & -1.7324 & -1.7325 & -1.7326 & -0.0001 \\
\hline & 2 & +0.9485 & +1.3245 & +1.3256 & +1.3245 & -0.0011 \\
\hline \multirow{5}{*}{1} & 0 & +2.3541 & +2.6541 & +2.6435 & +2.6435 & 0 \\
\hline & 0.5 & +1.4365 & +1.4563 & +1.4532 & +1.4653 & 0.0121 \\
\hline & 1 & +0.9 & +0.9632 & +0.9654 & +0.9568 & -0.0086 \\
\hline & 1.5 & -0.6862 & -0.6598 & -0.6548 & -0.6538 & 0.001 \\
\hline & 2 & +0.4756 & +0.4562 & +0.4562 & +0.4563 & 0.0001 \\
\hline
\end{tabular}

Table 1: The analytic results of $F(n)$, at different orders of HAM approximation compared with the numerical results, when $R e=1, t=1$ and $\varepsilon=1$.

\begin{tabular}{|c|c|c|c|c|c|c|}
\hline$r$ & $\eta$ & $9^{\text {th }}$ order & $10^{\text {th }}$ order & $11^{\text {th }}$ order & Numerical & Error \\
\hline \multirow{5}{*}{0} & 0 & +2.7032 & +2.6982 & +2.6921 & +2.6928 & 0.0007 \\
\hline & 0.5 & +1.1418 & +1.1824 & 1.1813 & +1.1832 & 0.0019 \\
\hline & 1 & -4.9328 & -4.9864 & -4.9862 & -4.9863 & -0.0001 \\
\hline & 1.5 & -4.5631 & -4.6382 & -4.6380 & -4.6382 & -0.0002 \\
\hline & 2 & +3.9732 & +3.8624 & +3.8614 & +3.8615 & 0.0001 \\
\hline \multirow{5}{*}{0.25} & 0 & +2.3289 & +2.3679 & +2.3576 & +2.3566 & -0.001 \\
\hline & 0.5 & +0.9631 & +1.0321 & +1.0322 & +1.0353 & 0.0031 \\
\hline & 1 & -3.7396 & -3.8921 & -3.8920 & -3.8933 & -0.0013 \\
\hline & 1.5 & -4.1382 & -4.3680 & -4.3670 & -4.3673 & -0.0003 \\
\hline & 2 & +4.9843 & +4.9621 & +4.9623 & +4.9636 & 0.0013 \\
\hline \multirow{5}{*}{0.5} & 0 & +2.3692 & +2.4692 & +2.4682 & +2.4683 & 0.0001 \\
\hline & 0.5 & +0.9328 & +0.9621 & +0.9620 & +0.9626 & 0.0006 \\
\hline & 1 & -2.7329 & -2.8392 & -2.8391 & -2.3966 & 0.4425 \\
\hline & 1.5 & -3.8321 & -3.8321 & -3.8323 & -3.8325 & -0.0002 \\
\hline & 2 & +2.6328 & +2.8628 & +2.8329 & +2.8629 & 0.03 \\
\hline \multirow{5}{*}{0.75} & 0 & +2.2836 & +2.2936 & +2.2937 & +2.2938 & 0.0001 \\
\hline & 0.5 & +0.9325 & +0.9626 & +0.9627 & +0.9628 & 0.0001 \\
\hline & 1 & -1.6312 & -1.6432 & -1.6433 & -1.6432 & 0.0001 \\
\hline & 1.5 & -2.3895 & -2.6892 & -2.6893 & -2.6896 & -0.0003 \\
\hline & 2 & +0.9865 & +0.9625 & +0.9623 & +0.9626 & 0.0003 \\
\hline \multirow{5}{*}{1} & 0 & +2.3286 & +2.4621 & +2.4620 & +2.4626 & 0.0006 \\
\hline & 0.5 & +0.7283 & +0.9621 & +0.9620 & +0.9622 & 0.0002 \\
\hline & 1 & -0.8325 & -0.8217 & -0.8218 & -0.8319 & -0.0101 \\
\hline & 1.5 & +0.8176 & +0.9163 & +0.9162 & +0.9163 & 0.0001 \\
\hline & 2 & +1.9086 & +1.9321 & +1.9323 & +1.9326 & 0.0003 \\
\hline
\end{tabular}

Table 2: The analytic results of $H(\eta)$ at different orders of HAM approximation compared with the numerical results, when $\operatorname{Re}=1, t=1$ and $\varepsilon=1$.
Figure 5, shows that, the time variation in the radial direction is obtained by the $11^{\text {th }}$ order approximation of the HAM for fixed values of $\operatorname{Re}=1, \epsilon=1$ and $\eta=0: 6$ with the different values of $\gamma$ the fluid is moving downward near the bottom wall. And the value of $\gamma$ coincide at $t=3$, at this time the flow will become steady and after this time the value of $\gamma$ increases and the fluid is moving near to the top wall.

From Figure 6, we observe that the time variation in the radial direction is obtained by the $11^{\text {th }}$ order approximation of the HAM for fixed values of $\operatorname{Re}=1, \epsilon=1$ and $\eta=0: 6$ with the different values of $\gamma$. When increasing the value of the fluid is moving downward near the bottom wall. In this figure also the fluid velocity coincide at $t=3$, so that the flow will become steady at this time. After this the value of the fluid velocity increases and the fluid is moving upward to the top wall. Figures 7 and 8 , shows that the velocity pro les in the radial direction under two values of Reynolds number are illustrated for different values of $\gamma=0$ and $\gamma=1$ by keeping time and amplitude as constant. It is obvious that with increasing Re, the velocity pro les get closer to the bottom wall. The maximum negative velocity decreases with increasing value of Re.

Figures 9 and 10, shows that the time variation in the radial direction and vertical direction is obtained by setting $\operatorname{Re}=1, \eta=0: 6, t=1$ and $\gamma=0: 5$ with the different values of amplitude $(\epsilon)$ in the $11^{\text {th }}$ order approximation of HAM. For increasing value of $\epsilon$, the fluid is moving near to the top wall with an out flow in the radial direction near the lower disk. In this case the fluid does not become steady because of the

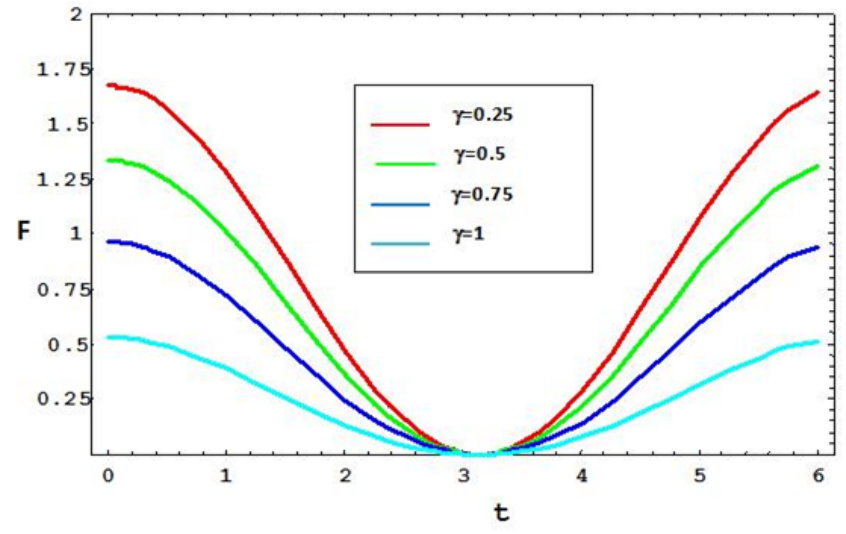

Figure 5: The time variation in the radial direction obtained by the 11th order approximation of the HAM when $\epsilon=1, t=1$ and $R e=1$.

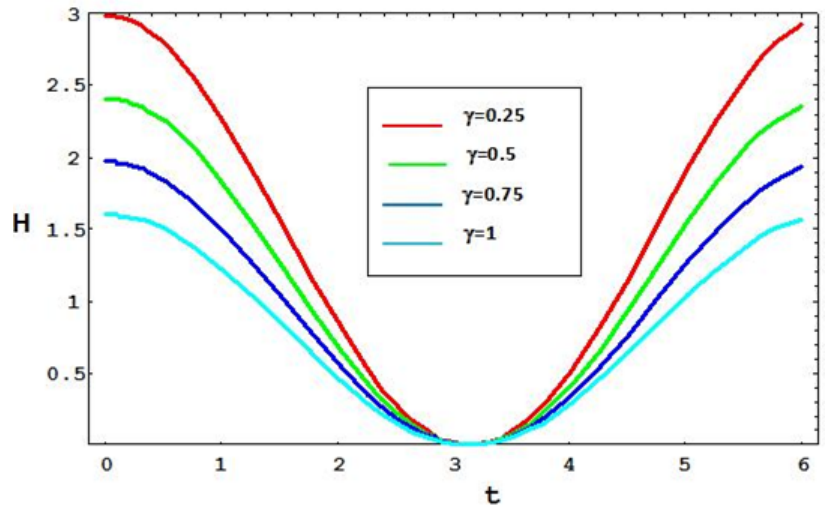

Figure 6: The time variation in the vertical direction obtained by the 11th orde approximation of the HAM when $\epsilon=1, t=1$ and $R e=1$. 


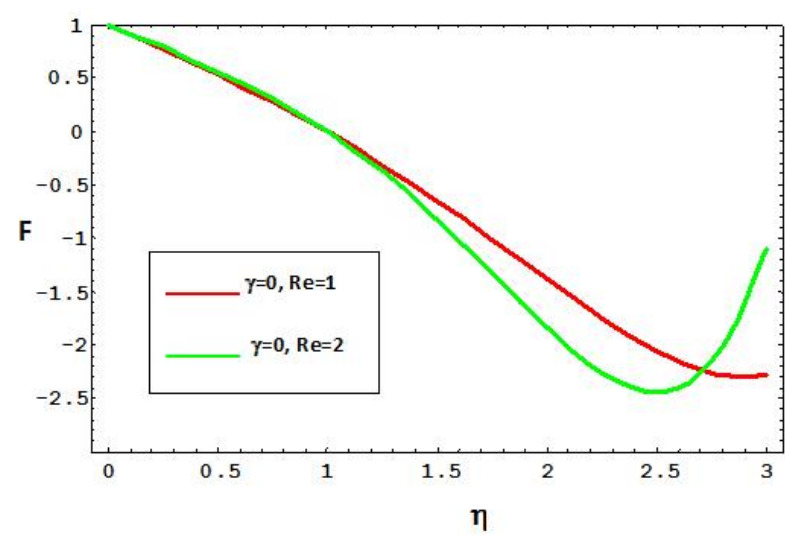

Figure 7: The velocity profile in the radial direction when $€=1$ and $t=1$

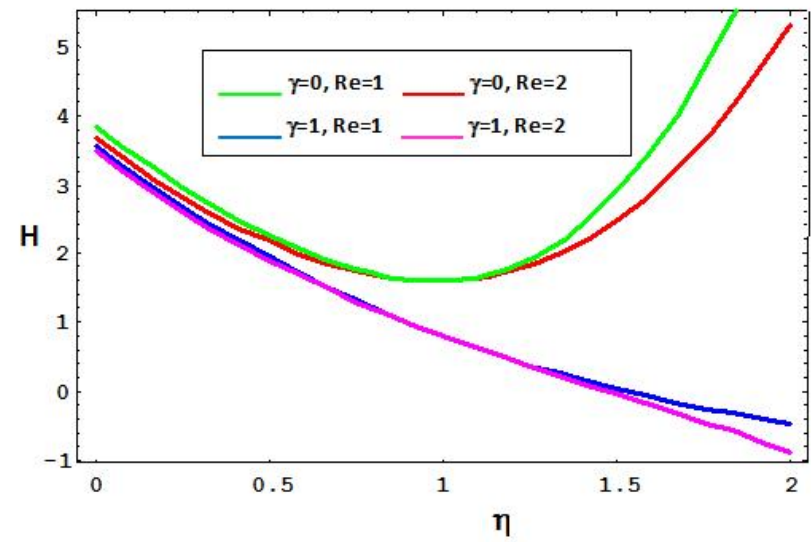

Figure 8: The velocity profile in the vertical direction when $\epsilon=1$ and $t=1$.

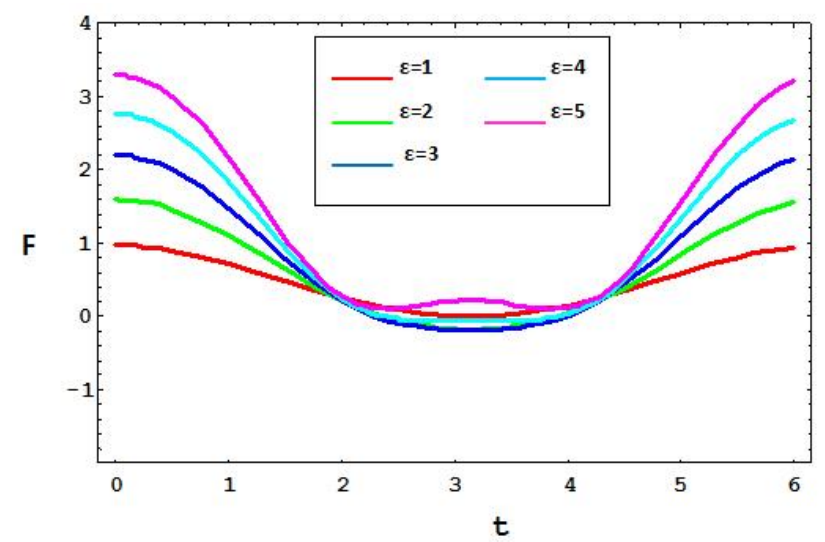

Figure 9: The time variation in the radial direction when $\mathrm{Re}=1, \mathrm{y}=0.5, \mathrm{n}=0.6$ and $\mathrm{t}=1$.

variation of amplitude in $\mathrm{t}$ direction.

\section{Conclusion}

In this paper, the HAM was used for finding the analytic solutions of the system of nonlinear ODE derived from von Karman type similarity transform for the unsteady state three dimensional

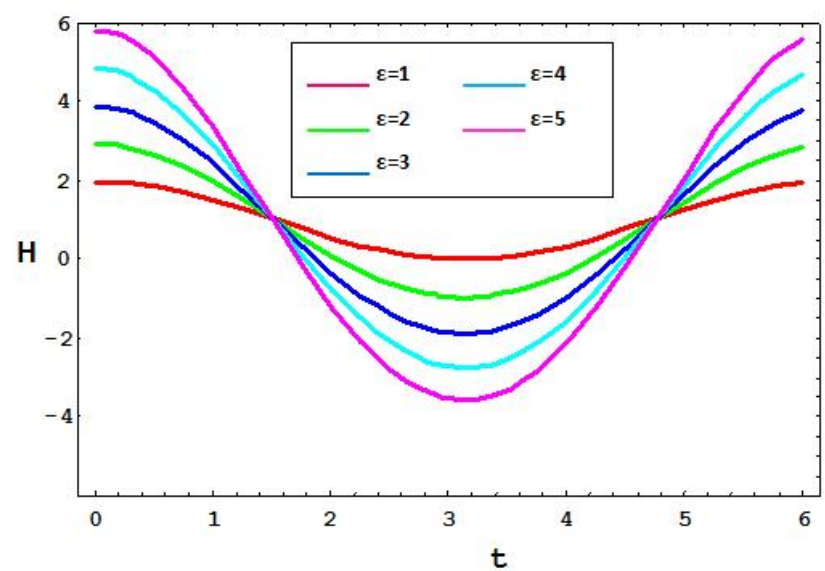

Figure 10: The time variation in the vertical direction when $\operatorname{Re}=1, y=0.5, \eta=0.6$ and $\mathrm{t}=0$.

Navier-Stokes equations of flow between two parallel disks. Unlike perturbation methods, the HAM does not depend on any small physical parameters. Thus homotopy analysis method is valid for both weakly and strongly nonlinear problems. Different from all other analytic methods, the homotopy analysis method provides us a simple way to adjust and control the convergence region of the series solution by means of auxiliary parameter $\hbar$. Thus, the auxiliary parameter $\hbar$ plays a vital role within the frame of HAM which can be determined by the $\hbar$ curves. The solution obtained by HAM is an infinite power series for appropriate initial approximation, which can be expressed in closed form. Continuously, the present success of the homotopy analysis method for nonlinear problem of flow between two parallel disks verifies that the method is a useful tool for nonlinear problems in many fields.

\section{References}

1. Nayfeh $A H$ (1993) Introduction to perturbation techniques. Wiley, New York.

2. Liao SJ (2003) Beyond Perturbation: Introduction to homotopy analysis method Chapman and Hall, CRC Press, Boca Raton.

3. He JH (2006) Homotopy Perturbation Method for solving boundary value problems. Physics Letters A 350: 87-88.

4. Allan FM (2007) Derivation of the Adomain Decomposition Method using Homotopy Analysis Method. Journal of Computer and Applied Mathematics 190: 6-14.

5. Dinarvand S, Rashidi MM, Shahmohamadi H (2009) Analytic approximate solution of three dimentional Navier-Stokes equations of flow between two streatchable disks. Wiley online library, Wiley periodicals 26: 1594-1607.

6. Liao SJ (2003) On the analytic solution of magne to hydrodynamic flows of non-Newtonian fluids over a stretching sheet. Journal of Fluid Mechanics 488 189-212.

7. Liao SJ (2004) On the homotopy analysis for nonlinear problems. Journal of Computer and Applied Mathematics 147: 499-513.

8. Liao SJ (2005) Comparison between the homotopy analysis method and Homotopy Perturbation Method. Journal of Computer and Applied Mathematics 169: 1186-1194.

9. Abbasbandy $S$ (2006) The application of homotopy analysis method to nonlinear equations arising in heat transfer. Physics Letters A 360: 109-116.

10. Allan FM, Syam MI (2005) On the analytic solution of non-homogeneous Blasius problem. Journal of Computer and Applied Mathematics 182: 362-371.

11. Allan FM (2009) Constructions of analytic solution to chaotic dynamical systems using the homotopy analysis method. Chaos Solitons Fractals 39: 1744-1752. 
Citation: Selvarani S, Nithyadevi N (2015) HAM Solution of an Unsteady Three Dimensional Navier-Stokes Equations of Flow between Two Parallel Disks. J Appl Computat Math 4: 256. doi:10.4172/2168-9679.1000256

12. Bouremel $Y$ (2007) Explicit series solution for the Glauert-jet problems by means of homotopy analysis method. Communications in Nonlinear Science Numerical Simulation 12: 714-724.

13. Ganji DD, Hosseini MJ, Shayegh J (2007) Some non-linear heat transfer equations solved by three approximate methods. International Journal of Heat Mass Transfer 34: 1003-1016.

14. Hayat T, Ahmad N, Sajid M, Asghar S (2007) On the MHD flow of a second grade fluid in a porous channel. Journal of Computer and Applied Mathematics 54: 407-414.

15. Hayat T, Sajid M, Ayub M (2007) A note on series solution for generalized Couette flow. Communications in Nonlinear Science Numerical Simulation 12: 1481-1487.

16. He JH (2000) A coupling method for homotopy technique and perturbation technique for non-linear problems. International Journal of Mechanics 35: $37-43$.

17. Ibrahim MO, Egbetade SA (2013) On the homotopy analysis method for an seir tuberculosis model. American Journal of Applied Mathematics and Statistics 4: $71-75$

18. Munawar S, Ali A, Saleem N, Naqeep A (2014) Swirling flow over an oscillatory stretchable disk. Journal of Mechanics 339-347.

19. Nave O, Lehavi Y, Dshtein VG (2012) Application of the HPM and HAM to the problem of the thermal explosion in a radiation gas with polydisperse fuel spray. Journal of Applied and Computational Mathematics.

20. Rafei M, Ganji DD, Daniali H (2007) Solution of the epidemic model by homotopy perturbation method. Journal of Computer and Applied Mathematics 187: 1056-1062.
21. Rafei M, Daniali H, Ganji DD (2007) Variational iteration method for solving the epidemic model and the prey and predator problem. Journal of Computer and Applied Mathematics 186: 1701-1709.

22. Ran XJ, Zhu QY, Li Y (2009) An explicit series solution of the squeezing flow between two in finite plates by means of the homotopy analysis method. Communications in Non-linear Science Numerical Simulation 14: 119-132.

23. Rand RH, Armbruster D (1987) Perturbation methods, bifurcation theory and computer algebraic. Applied Mathematical Sciences, Springer-Verlag, New York.

24. Rashidi MM, Domairry G, Dinarvand S (2009) Approximate solutions for the Burger and regularized long wave equations by means of the homotopy analysis method. Communications in Nonlinear Science Numerical Simulation 14: 708-717.

25. Tsai CC (2012) Homotopy method of fundamental solutions for solving certain nonlinear partial differential equations. Engineering Analysis with Boundary Elements 36: 1226-1234.

26. White FM (1991) Viscous fluid flow (2ndedn), McGraw-Hill, New York.

27. Ziabakhsh Z, Domairry G (2009) Solution of the laminar viscous flow in a semiporous channel in presence of a uniform magnetic field by using the homotopy analysis method. Communications in Nonlinear Science Numerical Simulation 14: $1284-1294$.

28. Zurigat M, Momani S, Alawneh A (2013) The multistage HAM: Application to a bio-chemical reaction model of fractional order. International Journal of Computer Mathematics 91: 1030-1040. 